\title{
Size Control of Mesoscale Aqueous Assemblies of Quantum Dots and Block Copolymers
}

\section{Supporting Information}

Huda Yusuf, Whan-Gi Kim, ${ }^{\dagger}$ Dong Hoon Lee, ${ }^{\dagger}$ Yunyong Guo and Matthew G. Moffitt*

Department of Chemistry, University of Victoria, P.O. Box 3065, Victoria, BC V8W 3V6 Canada

$\dagger$ Present Address: Department of Applied Chemistry, Konkuk University, 322 Danwol, Chungju, Chungbuk, Korea 380-220

*To Whom Correspondence Should be Addressed

E-mail:mmoffitt@uvic.ca 


\section{Photoluminescence Emission Spectra of PS300-CdS in Toluene for Different}

Excitation Wavelengths. Along with the emission spectrum reported in Figure 1a, for which the excitation wavelength was $\lambda_{\mathrm{ex}}=400 \mathrm{~nm}$, we investigated the emission spectra of PS300-CdS in toluene for various excitation wavelengths (Figure S1). It is evident that the position and shape of both band edge $(\sim 450 \mathrm{~nm})$ and trap state $(\sim 600 \mathrm{~nm})$ emissions are unchanged over the range of excitation wavelengths $\lambda_{\mathrm{ex}}=350-425 \mathrm{~nm}$, indicating a relatively narrow QD size distribution. The emission intensities increase with decreasing excitation wavelengths, as expected from the shape of the QD absorbance spectrum (Figure S1).

Static Light Scattering (SLS) of PS300-CdS in DMF. SLS of PS300-CdS in DMF was carried out to determine the molecular weight and radius of gyration of block copolymer-stabilized CdS nanoparticles before secondary self-assembly to form QDCMs. The SLS experiments were carried out on a Brookhaven Instruments multi-angle system equipped with a BI-200SM goniometer, a BI-9000AT digital autocorrelator, and a Melles Griot He-Ne Laser $(632.8 \mathrm{~nm})$ with maximum power output of $75 \mathrm{~mW}$. To ensure the accuracy of SLS measurement, great care was taken to eliminate dust from the samples. The stock solutions of PS300-CdS with concentration of ca. $10 \mathrm{mg} / \mathrm{mL}$ were prepared the night before SLS measurements to ensure equilibration. Spectroscopic grade DMF was filtered through two membrane filters with $0.20 \mu \mathrm{m}$ nominal pore size connected in series, and stock solutions of PS300-CdS colloid dispersed in DMF was filtered through two membrane filters with $0.45 \mu \mathrm{m}$ nominal pore size connected in series. All scintillation vials were thoroughly cleaned with filtered DMF, and stock solutions of the 
PS300-CdS were filtered into the dust-free scintillation vials. Successive dilutions of the colloids were carried out by adding known quantities of filtered DMF. SLS measurements were carried out in a concentration range from $1-0.1 \mathrm{mg} / \mathrm{mL}$ and the angles of detection ranged from $15-155^{\circ}$ with 5 degree increments between measurements. Ten repeat measurements of scattered light intensity were taken at each angle and concentration. The reported aggregation number and radius of gyration were determined for the average results of two separate Zimm plots obtained from different stock solutions. All SLS measurements were conducted at $23^{\circ} \mathrm{C}$.

For a Zimm plot, ${ }^{1,2}$ scattering intensity data is plotted according to the Zimm equation for light scattering from polymer solutions:

$\frac{K c}{\Delta R_{\theta}}=\frac{1}{M_{w} P(\theta)}+2 A_{2} c$

where $R_{\theta}$ is the excess Rayleigh scattering ratio, $P(\theta)$ is the particle scattering factor, $M_{\mathrm{w}}$ is the weight-average molar mass of the sample, $A_{2}$ is the second virial coefficient, $c$ is the polymer concentration and $K$ is a composite of optical and fundamental constants. The excess Rayleigh ratio is the ratio of light scattered from the solution in excess of solvent scattering at an angle of detection $\theta, I_{\theta}$, with respect to the incident beam intensity, $I_{0}: R_{\theta}=k I_{\theta} I_{0}$, where $k$ is an instrumental constant. In the typical Zimm plot approach, $M_{\mathrm{w}}$ is analyzed by detecting scattered light for each concentration at a series of angles, followed by extrapolation to $\theta=0$, where the scattering form factor $P(\theta)=1$, and to $c=0$, where the second term on the r.h.s. of Equation 1 becomes negligible. 
A typical Zimm plot obtained for PS300-CdS in DMF is shown in Figure S2. Based on the average of two runs, we determined $M_{w}=1.78 \pm 0.06 \times 10^{6} \mathrm{~g} / \mathrm{mol}$, from which an aggregation number of $54 \pm 2$ copolymer chains surrounding each CdS QD was determined. Also from the average of the two Zimm plots, the radius of gyration, $r_{\mathrm{g}}$, of PS300-CdS in DMF was determined to be $21 \pm 8 \mathrm{~nm}$.

Dynamic Light Scattering (DLS) of PS300-CdS in DMF. DLS of PS300-CdS in DMF was carried out to characterize the hydrodynamic diameter $d_{\mathrm{h}, \mathrm{PS}-\mathrm{CdS}}$ of the block copolymer-stabilized CdS nanoparticles before secondary self-assembly to form QDCMs. The $d_{\mathrm{h}, \mathrm{PS}-\mathrm{CdS}}$ value for PS300-CdS in DMF was determined by analyzing the autocorrelation function using the method of cumulants for various scattering angles and

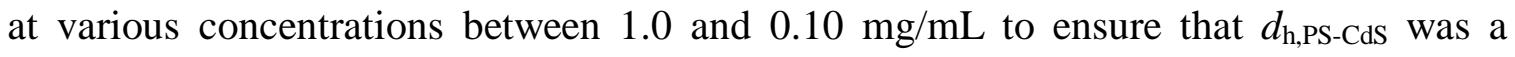
"true" $z$-average size, independent of interparticle correlations.

\section{Basic Theory of DLS}

Dynamic light scattering (DLS) allows the detection of quick changes in the scattered light intensity caused by solution density changes. These changes are a result of Brownian motion of particles in solution, and can be investigated to determine the diffusion coefficient, $D_{0}$, of the particles. Using the Stokes-Einstein relation, the diffusion coefficient can subsequently be used to calculate the hydrodynamic diameter $\left(d_{\mathrm{h}}\right)$, which is a measure of particle size in solution including any solvent molecules that move with the particle., 
The fundamental quantity measured in a DLS experiment is the intensity autocorrelation function, $G_{2}(\tau)$, which for a dilute solution of monodisperse particles is an exponentially decaying function of the delay time, $\tau$. The intensity autocorrelation function can be linked to the normalized electric field autocorrelation function, $g_{1}(\tau)$, in the following manner:

$G_{2}(\tau)=B\left(1+\beta\left|g_{1}(\tau)\right|^{2}\right)$

where $B$ is the measured baseline of the decaying autocorrelation function and $\beta$ is the variable consistency factor. If the population is monodisperse and the particles are noninteracting, $\left|g_{1}(\tau)\right|$ is also an exponential function and can be expressed as:

$\left|g_{1}(\tau)\right|=\exp (-\Gamma \tau)$

where $\Gamma$ is the relaxation rate of the autocorrelation function with units of $\mathrm{s}^{-1} . \Gamma$ can be related to the diffusion coefficient, $D$, as follows:

$\Gamma=D q^{2}$

where $q$ is the scattering vector and is expressed as:

$q=(4 \pi n / \lambda) \sin (\theta / 2)$ 
where $n$ is the refractive index of the solvent, $\lambda$ is the wavelength of the incident light, and $\theta$ is the scattering angle.

It is usually observed that the experimental autocorrelation function is not a single exponential function and that there is a distribution of relaxation rates due to a distribution of particle sizes in solution. Data were fitted by the method of cumulants developed by Koppel, using the Brookhaven correlator software. In this method, the autocorrelation function is expanded about a $z$-average relaxation rate, $(\bar{\Gamma})_{z}$. The result is a polynomial expansion in the delay time with cumulants (moments about $(\bar{\Gamma})_{z}$ ) as the restrictions to be fitted. For polydisperse, point-like, isotropic particles lacking intermolecular interactions the expansion may be written as follows:

$\left|g_{1}(\tau)\right|=\exp \left\{-\bar{\Gamma} \tau+(\mu / 2 !) \tau^{2}+\ldots\right\}$

where $\bar{\Gamma}$ is the mean relaxation rate and $\mu$ is the second moment of the distribution of relaxation rates. The ratio $\mu /(\bar{\Gamma})_{z}^{2}$ is related to the size polydispersity of the sample. Using $(\bar{\Gamma})_{z}$, measured at various angles, a $z$-average effective diffusion coefficient, $D_{\mathrm{T}}$, is determined from Equation 4, then an effective $z$-average hydrodynamic diameter, $d_{\mathrm{h}, \mathrm{eff}}$, is calculated using the Stokes-Einstein equation:

$D_{\mathrm{T}}=k_{\mathrm{B}} T / 3 \pi \eta d_{\mathrm{h}, \mathrm{eff}}$

where $k_{\mathrm{B}}$ is Boltzmann's constant, $T$ is the temperature, and $\eta$ is the viscosity of the solvent. Due to frictional and thermodynamic interparticle interactions, the effective 
hydrodynamic diameter $d_{\mathrm{h} \text {,eff }}$ is not always equal to the true hydrodynamic diameter for an individual non-interacting particle, $d_{\mathrm{h}}$. To determine $d_{\mathrm{h}}$, the effective diffusion coefficient is measured from cumulant analysis for solutions of various concentrations and then extrapolated to infinite dilution $(c=0)$ to determine the true diffusion coefficient, $D_{0} . \quad D_{0}$ is then used to calculate $d_{\mathrm{h}}$ using the Stokes-Einstein relation (Equation 7).

\section{$\underline{\text { DLS Measurements }}$}

To ensure the accuracy of DLS measurements, great care was taken to eliminate dust from the samples. For DLS measurements of PS300-CdS, spectroscopic grade DMF was filtered through 2 membrane filters connected in series with $0.20 \mu \mathrm{m}$ nominal pore size; stock solutions of PS300-CdS colloids dispersed in DMF with concentration of ca. 5 $\mathrm{mg} / \mathrm{mL}$ were filtered through 2 membrane filters connected in series with $0.45 \mu \mathrm{m}$ nominal pore size. All scintillation vials were thoroughly cleaned with filtered DMF, and the stock solution of the PS300-CdS colloids was filtered into the dust-free scintillation vials. Successive dilutions of the colloids were carried out by adding known quantities of filtered DMF. DLS measurements were conducted at these angles to determine the true hydrodynamic diameter: $35^{\circ}, 50^{\circ}, 70^{\circ}, 90^{\circ}$, and $120^{\circ}$ and at five different concentrations in the range of $1.0-0.1 \mathrm{mg} / \mathrm{mL}$. For each experiment, 3 repeat measurements of the autocorrelation function were obtained. All DLS measurements were conducted at $23^{\circ} \mathrm{C}$.

The cumulant method was used to analyze the normalized electric field autocorrelation function, in order to determine a mean relaxation rate, $\bar{\Gamma}$, for each

scattering angle. Figure S3a shows plots of $\bar{\Gamma}$ vs. the square of the scattering vector $q^{2}$ 
for various concentrations. Each of these plots is linear, consistent with Equation 4, which indicates a diffusive relaxation mode from the free translational diffusion of particles. From the slope of each line, the effective diffusion coefficient $D_{\mathrm{T}}$ was determined for each concentration. Figure S3b shows $D_{\mathrm{T}}$ plotted against concentration and the resulting extrapolation to infinite dilution $(c=0)$ with the diffusion coefficient at infinite dilution $D_{0}=1.55 \pm 0.02 \times 10^{-7} \mathrm{~nm}^{2} \mathrm{~s}^{-1}$ determined from the intercept. We see that there is a linear relationship between $D_{\mathrm{T}}$ and $c$ with a positive slope giving $k_{\mathrm{D}}=1.6 \pm 0.2$ x $10^{-4} \mathrm{~cm}^{3} \mathrm{~g}^{-1}\left(D_{\mathrm{T}}=D_{0}\left(1+k_{\mathrm{D}} c\right)\right)$. From the diffusion coefficient $D_{0}$, the hydrodynamic diameter at infinite dilution, $d_{\mathrm{h}}$, was calculated using the Stokes-Einstein relation and a value of $36 \mathrm{~nm}$ was obtained.

Details of Calculation of $n_{\mathrm{PS}-\mathrm{CdS}}$, the Number of PS300-CdS Particles per QDCM, from the QDCM Core Diameter, $\boldsymbol{d}_{\mathbf{c}}$. From the tunable QDCM particle sizes determined from TEM, $d_{\mathrm{c}}$, for each initial polymer concentration, we calculated the average number of PS300-CdS particles per QDCM, $n_{P S-C d S}$, in the following manner. The average volume of a QDCM core (i.e. excluding the PAA chains on the surface), $V_{c}$, is determined from:

$V_{c}=\frac{4}{3} \pi r_{c}^{3}$

where $r_{c}=d_{c} / 2$ is the QDCM core radius. 
From $V_{\mathrm{c}}$, the mass of a QDCM core, $m_{\mathrm{c}}$, is determined assuming a density of $\sim 1 \mathrm{~g} / \mathrm{mL}$ for the core (approximately the bulk density of PS):

$m_{\mathrm{c}}=V_{\mathrm{c}} \times 1 \mathrm{~g} / \mathrm{cm}^{3}$

The total QDCM core mass is the sum of two components: 1) the mass of PS blocks from the PS(665)- $b$-PAA(68) stabilizing chains, $m_{\mathrm{c}, \mathrm{sc}}$, and 2) the mass of aggregated PS300-

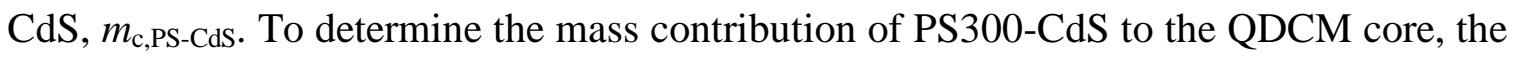
total core mass is multiplied by 0.52 , which is the weight fraction of PS300-CdS within the core, given that the total weight fraction of PS300-CdS within the blend is $f=0.50$, assuming a statistical distribution of stabilizing chains over all QDCM particles. The small difference in these weight fractions is due to the fact that only the PS blocks of stabilizing chains are included in the mass of the QDCM core:

$m_{\mathrm{c}, \mathrm{PS}-\mathrm{CdS}}=m_{\mathrm{c}} \times 0.52$

To determine the mass of a single PS300-CdS particle, we use the weight-average molecular weight of PS300-CdS determined from static light scattering (Table 1):

$m_{\text {PS-CdS }}=M_{\mathrm{w}} / N_{\mathrm{A}}=1.78 \times 10^{6} \mathrm{~g} / \mathrm{mol} / 6.02 \times 10^{23}$ particles $/ \mathrm{mol}=2.96 \times 10^{-18} \mathrm{~g}$ 
Finally, the number of PS300-CdS particles per QDCM is determined by dividing the total mass of PS300-CdS particles per QDCM, $m_{\mathrm{c}, \mathrm{PS}-\mathrm{CdS}}$, by $m_{\mathrm{PS}-\mathrm{CdS}}$ :

$n_{\text {PS-CdS }}=m_{c, \text { PS-CdS }} / m_{\text {PS-CdS }}=m_{c, \text { PS-CdS }} / 2.96 \times 10^{-18} \mathrm{~g}$

Combining Equations 8-12, we obtain:

$$
\begin{aligned}
& n_{\text {PS-CdS }}=\left[4 / 3 \times \pi \times\left(d_{\mathrm{c}} / 2\right)^{3} \times 1 \mathrm{~g} / \mathrm{cm}^{3} \times 0.52\right] / 2.96 \times 10^{-18} \mathrm{~g}=9.1984 \times 10^{16} \mathrm{~cm}^{-3} \times d_{\mathrm{c}}^{3} \\
& \text { or } \\
& n_{\text {PS-CdS }}=9.1984 \times 10^{-5} \mathrm{~nm}^{-3} \times d_{\mathrm{c}}^{3}
\end{aligned}
$$

\section{Details of Calculation of the PAA Surface Density, $\sigma_{\mathrm{PAA}}$, from the QDCM}

Core Diameter, $\boldsymbol{d}_{\mathbf{c}}$. The surface density of PAA chains, $\sigma_{\mathrm{PAA}}$, was also calculated from $d_{\mathrm{c}}$ for each initial polymer concentration. As in the calculation of $n_{\mathrm{PS}-\mathrm{CdS}}$, Equations 8 and 9 above were used to determine the total mass of the QDCM core, $m_{\mathrm{c}}$. To determine the mass contribution of stabilizing chains to the QDCM core, the total core mass is multiplied by 0.48 , which is the weight fraction of PS blocks from the stabilizing chains within the core, given that the total weight fraction of PS300-CdS within the blend is $f=$ 0.50 , assuming a statistical distribution of stabilizing chains over all QDCM particles:

$m_{\mathrm{c}, \mathrm{sc}}=m_{\mathrm{c}} \times 0.48$ 
The number of stabilizing chains per QDCM particle, $n_{\mathrm{sc}}$, is then calculated by dividing the mass contribution of PS blocks from stabilizing chains to the QDCM core, $m_{\mathrm{c}, \mathrm{sc}}$, by the mass of each PS block, $m_{\mathrm{sc}, \mathrm{PS}}$ :

$m_{\mathrm{sc}, \mathrm{PS}}=665$ styrene units $\times 104.15 \mathrm{~g} / \mathrm{mol} / 6.02 \times 10^{23} \mathrm{~mol}^{-1}=1.15 \times 10^{-19} \mathrm{~g}$

$n_{\mathrm{sc}}=m_{\mathrm{c}, \mathrm{sc}} / m_{\mathrm{sc}, \mathrm{PS}}=m_{\mathrm{c}, \mathrm{sc}} / 1.15 \times 10^{-19} \mathrm{~g}$

The number of stabilizing chains per QDCM particle must equal the number of PAA chains at the QDCM surface. The surface area per QDCM core is first determined from:

$A_{\mathrm{c}}=4 \pi\left(d_{\mathrm{c}} / 2\right)^{2}$

And finally $\sigma_{\mathrm{PAA}}$ is determined as follows:

$\sigma_{\mathrm{PAA}}=n_{\mathrm{sc}} / A_{\mathrm{c}}$

Combining Equations 8-9 and 14-18 we obtain:

$$
\begin{aligned}
& \sigma_{\mathrm{PAA}}=\left\{\left[4 / 3 \times \pi \times\left(d_{\mathrm{c}} / 2\right)^{3} \times 1 \mathrm{~g} / \mathrm{cm}^{3} \times 0.48\right] / 1.15 \times 10^{-19} \mathrm{~g}\right\} / 4 \pi\left(d_{\mathrm{c}} / 2\right)^{2}= \\
& 6.9565 \times 10^{17} \mathrm{~cm}^{-3} \times d_{\mathrm{c}}=6.9565 \times 10^{-4} \mathrm{~nm}^{-3} \times d_{\mathrm{c}}
\end{aligned}
$$




\section{References}

1) Zimm, B. H. J. Chem. Phys. 1948, 16, 1099.

2) Cowie, J. M. G. Polymers: Chemistry and Physics of Modern Materials. $2^{\text {nd }}$ Edition, Ed., Nelson Thornes, 1991.

3) Brookhaven Instruments Instruction Manual for BI-9000AT Digital Autocorrelator, Brookhaven Instruments Coorporation, 2003.

4) Zhang, L.; Barlow, R. J.; Eisenberg, A. Macromolecules 1995, $28,6055$.

\section{Supporting Information Figure Captions}

Figure S1. Absorbance and photoluminescence (PL) spectra of PS300-CdS dissolved in spectroscopic grade toluene $\left(\lambda_{\mathrm{ex}}=350,375,400\right.$, and $\left.425 \mathrm{~nm}\right)$. The core size of the CdS was determined to be $d_{\mathrm{CdS}}=4.0 \mathrm{~nm}$ from $\lambda_{\text {thresh }}=465 \mathrm{~nm}$. The PL spectrum was recorded at $1 \mathrm{~nm}$ spectral resolution using a $425 \mathrm{~nm}$ filter for the first three excitations and $515 \mathrm{~nm}$ for the last excitation and was solvent-subtracted.

Figure S2. Representative Zimm plot from static light scattering (SLS) data of PS300$\mathrm{CdS}$ in DMF. The angles used in these measurements were from $5^{\circ}$ to $155^{\circ}$ in $5^{\circ}$ increments, with concentrations between 0.1 to $1.0 \mathrm{mg} / \mathrm{mL}$.

Figure S3. Dynamic light scattering (DLS) data for PS300-CdS dissolved in DMF: a) $\bar{\Gamma}$ vs $q^{2}$ for various concentrations of PS300-CdS in DMF; b) plot of the effective diffusion coefficient $D_{\mathrm{T}}$ vs concentration. 